\title{
The nonmonotonic Gentzen deduction systems for the propositional logic
}

\author{
Cungen Cao, Yuefei Sui, Yuhui Wang \\ Key Laboratory of Intelligent Information Processing, Institute of Computing Technology, Chinese Academy of Sciences, China
}

Received: February 15, 2016

Accepted: April 24, 2016

Online Published: August 22, 2016

DOI: $10.5430 /$ air.v5n2p111

URL: http://dx.doi.org/10.5430/air.v5n2p111

\begin{abstract}
The traditional propositional logic is monotonic. With the same logical language as, the same valuation as and the validity of a sequent different from the traditional propositional logic, a propositional logic could be nonmonotonic. In this paper, the four Gentzen deduction systems $G^{1}, G^{2}, G^{3}, G^{4}$ and their dualities $G_{1}, G_{2}, G_{3}, G_{4}$ will be given which are proved to be sound and complete with respect to the four definitions and their dualities of the validity of sequents, among which one is traditional and others are variations of the traditional one. Moreover, $G^{1}, G^{3}$ are monotonic in both $\Gamma$ and $\Delta$; and $G^{2}, G^{4}$ are monotonic in $\Gamma$ and nonmonotonic in $\Delta$. Dually, $G_{1}, G_{3}$ are nonmonotonic in both $\Gamma$ and $\Delta$; and $G_{2}, G_{4}$ are nonmonotonic in $\Gamma$ and monotonic in $\Delta$.
\end{abstract}

Key Words: Propositional logic, The Gentzen deduction system, Validity, Sequent, Soundness, Completeness

\section{INTRODUCTION}

The traditional Gentzen deduction system $G^{1}$ for the propositional logic is monotonic, ${ }^{[1,2]}$ that is, given any theories $\Gamma, \Gamma^{\prime}, \Delta, \Delta^{\prime}$, if sequent $\Gamma \Rightarrow \Delta$ is provable in $G^{1}$ and $\Gamma \subseteq \Gamma^{\prime}, \Delta \subseteq \Delta^{\prime}$ then $\Gamma^{\prime} \Rightarrow \Delta, \Gamma \Rightarrow \Delta^{\prime}, \Gamma^{\prime} \Rightarrow \Delta^{\prime}$ are provable in $G^{1}$. Correspondingly, the Gentzen deduction system $G_{1}$ for $\Gamma \nRightarrow \Delta$ is nonmonotonic,${ }^{[3-5]}$ that is, given any theories $\Gamma, \Gamma^{\prime}, \Delta, \Delta^{\prime}$, if sequent $\Gamma \nRightarrow \Delta$ is provable in $G_{1}$ and $\Gamma \subseteq \Gamma^{\prime}, \Delta \subseteq \Delta^{\prime}$ then it may be true that $\Gamma^{\prime} \nRightarrow \Delta, \Gamma \nRightarrow \Delta^{\prime}, \Gamma^{\prime} \nRightarrow \Delta^{\prime}$ are provable in $G_{1}$.

The nonmonotonic logics are different from the monotonic logics in that the deduction is nonmonotonic. The traditional logics, such as the propositional logic, the first-order logic, modal logic, etc., are monotonic. The nontraditional logics, such as the default logic, the autoepistemic logic, circumscription, etc., are nonmonotonic..$^{[4-8]}$
The nonmonotonicity of a nonmonotonic logic follows from using a negation $\Delta \forall A$ of a monotonic deduction $\Delta \vdash A$. We found that each nonmonotonic logic has the occurrence of $\Delta \nvdash A$. For example, a formula $B$ is deducible in the default logic (or in some extension of default theory $\left(\Delta,\left\{\frac{A: B}{B}\right\}\right.$ ) from a default theory $\left(\Delta,\left\{\frac{A: B}{B}\right\}\right)$ if $A$ is deducible in propositional logic from $\Delta$ and $\neg B$ is not, that is,

$$
\Delta \vdash A \& \Delta \forall \neg \neg .
$$

It is obvious that the monotonicity of $\Delta \vdash A$ implies the nonmonotonicity of $\Delta \forall A$.

As a deduction relation, $\not \forall$ is contradictory to $\vdash$. Correspondingly, in the Gentzen deduction systems, the validity of $\Gamma \Rightarrow \Delta$ is contradictory to the invalidity of $\Gamma \Rightarrow \Delta$, i.e., the validity of $\Gamma \mapsto \Delta$, where $\Gamma \mapsto \Delta$ is valid if there is an

${ }^{*}$ Correspondence: Yuefei Sui; Email: yfsui@ict.ac.cn; Address: Key Laboratory of Intelligent Information Processing, Institute of Computing Technology, Chinese Academy of Sciences, China. 
assignment $v$ such that $v$ satisfies $\Gamma$ and does not satisfy $\Delta$, where $v$ satisfies $\Gamma$ if $v$ satisfies each formula in $\Gamma$; and $v$ satisfies $\Delta$ if $v$ satisfies some formula in $\Delta$.

Therefore, as a contradictory relation $\vdash \Gamma \mapsto \Delta$ of $\vdash \Gamma \Rightarrow \Delta$, there is a Gentzen-typed deduction system $G_{1}$ such that $G_{1}$ is sound and complete, that is, for any sequent $\Gamma \mapsto \Delta$, if $\Gamma \mapsto \Delta$ is provable in $G_{1}$ then $\Gamma \mapsto \Delta$ is valid; and conversely, if $\Gamma \mapsto \Delta$ is valid then $\Gamma \mapsto \Delta$ is provable in $G_{1}$.

Formally, the validity of sequent $\Gamma \Rightarrow \Delta$ is defined as follows:

$\models G^{1} \Gamma \Rightarrow \Delta$ if for any assignment $v, v \models \Gamma$ implies

$v \models \Delta$, where $v \models \Gamma$ if for every $A \in \Gamma, v(A)=1$; and

$v \models \Delta$ if for some $B \in \Delta, v(B)=1$.

Correspondingly, sequent $\Gamma \nRightarrow \Delta$ (denoted by $\Gamma \mapsto \Delta)$ being valid is defined as follows:

$\models_{G_{1}} \Gamma \Rightarrow \Delta$ if there is an assignment $v$ such that $v \models \Gamma$ and $v \not \forall \Delta$, where $v \models \Gamma$ if for every $A \in \Gamma, v(A)=1$; and $v \not \models \Delta$ if for every $B \in \Delta, v(B)=0$.

We consider other possible definitions of the validity and have the following four definitions:

- for any assignment $v, v \models \Gamma$ implies $v \models \Delta$, where $v \models \Gamma$ if for every $A \in \Gamma, v(A)=1$; and

$$
\left\{\begin{array}{l}
v \models \Delta \text { if for some } \mathrm{B} \in \Delta, v(B)=1 ; \\
v \models \Delta \text { if for every } \mathrm{B} \in \Delta, v(B)=1 ; \\
v \models \Delta \text { if for some } \mathrm{B} \in \Delta, v(B)=0 ; \\
v \models \Delta \text { if for every } \mathrm{B} \in \Delta, v(B)=0 ;
\end{array}\right.
$$

- there is an assignment $v$ such that $v \models \Gamma$ and $v \forall \forall$, where $v \models \Gamma$ if for every $A \in \Gamma, v(A)=1$; and

$$
\left\{\begin{array}{l}
v \not \models \Delta \text { if for every } \mathrm{B} \in \Delta, v(B)=0 ; \\
v \forall \forall \Delta \text { if for some } \mathrm{B} \in \Delta, v(B)=0 ; \\
v \not \models \Delta \text { if for every } \mathrm{B} \in \Delta, v(B)=1 ; \\
v \not \forall \Delta \text { if for some } \mathrm{B} \in \Delta, v(B)=1 .
\end{array}\right.
$$

Therefore, we have four Gentzen deduction systems $G^{1}, G^{2}, G^{3}, G^{4[2,9-11]}$ and their dualities $G_{1}, G_{2}, G_{3}, G_{4}$, where

$$
\begin{array}{l|l}
\text { definition } & \text { system } \\
\hline \forall v(\forall A \in \Gamma(v(A)=1) \Rightarrow \exists B \in \Delta(v(B)=1)) & \mathbf{G}^{1} \\
\forall v(\forall A \in \Gamma(v(A)=1) \Rightarrow \forall B \in \Delta(v(B)=1)) & \mathbf{G}^{2} \\
\forall v(\forall A \in \Gamma(v(A)=1) \Rightarrow \exists B \in \Delta(v(B)=0)) & \mathbf{G}^{3} \\
\forall v(\forall A \in \Gamma(v(A)=1) \Rightarrow \forall B \in \Delta(v(B)=0)) & \mathbf{G}^{4} ;
\end{array}
$$

and

\begin{tabular}{l|l} 
definition & system \\
\hline$\exists v(\forall A \in \Gamma(v(A)=1) \& \exists B \in \Delta(v(B)=1))$ & $\mathbf{G}_{4}$ \\
$\exists v(\forall A \in \Gamma(v(A)=1) \& \forall B \in \Delta(v(B)=1))$ & $\mathbf{G}_{3}$ \\
$\exists v(\forall A \in \Gamma(v(A)=1) \& \exists B \in \Delta(v(B)=0))$ & $\mathbf{G}_{2}$ \\
$\exists v(\forall A \in \Gamma(v(A)=1) \& \forall B \in \Delta(v(B)=0))$ & $\mathbf{G}_{1}$.
\end{tabular}

It will be proved that: ${ }^{[6,8,12-15]}$

(1) $G^{1}, G^{3}$ are monotonic in both $\Gamma$ and $\Delta$;

(2) $G^{2}, G^{4}$ are monotonic in $\Gamma$ and nonmonotonic in $\Delta$;

(3) $G_{1}, G_{3}$ are nonmonotonic in both $\Gamma$ and $\Delta$;

(4) $G_{2}, G_{4}$ are nonmonotonic in $\Gamma$ and monotonic in $\Delta$.

This paper is organized as follows: the next section gives the basic definitions in the propositional logic; the third section gives the Gentzen deduction system $G^{1}$ for the traditional propositional logic and $G_{1}$ for the nonmonotonic propositional logic; the fourth section gives the Gentzen deduction systems $G^{2}$ and $G_{2}$ and proves that they are sound and complete; the fifth section gives sound and complete Gentzen deduction systems $G^{3}$ and $G_{3}$ and analyzes their monotonicity; the sixth section lists the sound and complete Gentzen deduction systems $G^{4}$ and $G_{4}$; and the last section concludes the whole paper with the table of monotonicity of all the systems.

\section{The logical language of the PROPO- SITIONAL LOGIC}

The logical language of the propositional logic consists of the following symbols:

- propositional variables: $p_{0}, p_{1}, \ldots$;

- logical connectives: $\neg, \wedge, \vee$, and

- auxiliary symbols: $($,$) .$

A string $A$ of symbols is a formula if

$$
A::=p|\neg p| A_{1} \wedge A_{2} \mid A_{1} \vee A_{2} .
$$

The semantics of the propositional logic is given by an assignment $v$, a function from the propositional variables to $\{0,1\}$.

Given an assignment $v$, a formula $A$ is true in $v$, denoted by $v \models A$, if

$$
\begin{cases}v(p)=1 & \text { if } A=p \\ v(p)=0 & \text { if } A=\neg p \\ v \models A_{1} \& v \models A_{2} & \text { if } A=A_{1} \wedge A_{2} \\ v \models A_{1} \text { orv } \models A_{2} & \text { if } A=A_{1} \vee A_{2}\end{cases}
$$

where $\sim, \&$, or are symbols used in the meta-language, and correspondingly, $\neg, \wedge, \vee$ are the ones used in the language. Therefore, $v \not \models A_{1}$ can be represented as $\sim\left(v \models A_{1}\right)$.

A sequent $\delta$ is a pair $(\Gamma, \Delta)$, denoted by $\Gamma \Rightarrow \Delta$, where $\Gamma, \Delta$ are sets of formulas.

A literal $l$ is a propositional variable or the negation of a propositional variable. 


\section{THE PROPOSITIONAL LOGIC $G^{1}$}

A sequent $\Gamma \Rightarrow \Delta$ is valid, denoted by $\models_{G^{1}} \Gamma \Rightarrow \Delta$, if for any assignment $v, v(A)=1$ for every $A \in \Gamma$ implies $v(B)=1$ for some $B \in \Delta$.

The Gentzen deduction system $G^{1}$ consists of the following axioms and deduction rules:

- Axioms:

$$
\left(A_{\Rightarrow}\right) \frac{\operatorname{incon}(\Gamma) \text { or } \operatorname{incon}(\Delta) \text { or } \Gamma \cap \Delta \neq \emptyset}{\Gamma \Rightarrow \Delta}
$$

where $\Gamma, \Delta$ are sets of literals.

- Deduction rules:

$$
\begin{array}{ll}
\left(\Rightarrow \wedge_{1}^{L}\right) \frac{\Gamma, A_{1} \Rightarrow \Delta}{\Gamma, A_{1} \wedge A_{2} \Rightarrow \Delta} & \left(\Rightarrow \wedge^{R}\right) \frac{\Gamma \Rightarrow B_{1}, \Delta \Gamma \Rightarrow B_{2}, \Delta}{\Gamma \Rightarrow B_{1} \wedge B_{2}, \Delta} \\
\left(\Rightarrow \wedge_{2}^{L}\right) \frac{\Gamma, A_{2} \Rightarrow \Delta}{\Gamma, A_{1} \wedge A_{2} \Rightarrow \Delta} & \\
\left(\Rightarrow \vee^{L}\right) \frac{\Gamma, A_{1} \Rightarrow \Delta \Gamma, A_{2} \Rightarrow \Delta}{\Gamma, A_{1} \vee A_{2} \Rightarrow \Delta} & \left(\Rightarrow \vee_{1}^{R}\right) \frac{\Gamma \Rightarrow B_{1}, \Delta}{\Gamma \Rightarrow B_{1} \vee B_{2}, \Delta} \\
& \left(\Rightarrow \vee_{2}^{R}\right) \frac{\Gamma \Rightarrow B_{2}, \Delta}{\Gamma \Rightarrow B_{1} \vee B_{2}, \Delta}
\end{array}
$$

Theorem 3.1 (The soundness and completeness theorem). For any sequent $\Gamma \Rightarrow \Delta$,

$$
\vdash_{G^{1}} \Gamma \Rightarrow \Delta \text { iff } \models_{G^{1}} \Gamma \Rightarrow \Delta
$$

The propositional logic of $G_{1}$

Definition 3.2 A sequent $\Gamma \mapsto \Delta$ is valid, denoted by $\models_{G_{1}} \Gamma \mapsto \Delta$ if there is an assignment $v$ such that $v \models \Gamma$ and $v \models \Delta$, where $v \models \Gamma$ if for each $A \in \Gamma, v(A)=1$; and $v \models \Delta$ if for each $B \in \Delta, v(B)=0$.

A sequent $\Gamma \mapsto \Delta$ is not valid if $\Gamma \mapsto \Delta$ is unsatisfiable, i.e., there is no assignment $v$ such that $v \models \Gamma$ and $v \models \Delta$.

Lemma 3.3 Given two sets $\Gamma, \Delta$ of literals, $\models_{G_{1}} \Gamma \mapsto \Delta$ if and only if $\Gamma$ and $\Delta$ are consistent, and $\Gamma \cap \Delta=\emptyset$.

The Gentzen deduction system $G_{1}$ consists of the following axioms and deduction rules:

- Axioms:

$$
\left(A_{\mapsto}\right) \frac{\operatorname{con}(\Gamma) \& \operatorname{con}(\Delta) \& \Gamma \cap \Delta=\emptyset}{\Gamma \mapsto \Delta}
$$

where $\Delta, \Gamma$ are sets of literals.

- Deduction rules:

$$
\begin{array}{ll}
\left(\mapsto \wedge^{L}\right) \frac{\Gamma, A_{1} \mapsto \Delta \Gamma, A_{2} \mapsto \Delta}{\Gamma, A_{1} \wedge A_{2} \mapsto \Delta} & \left(\mapsto \wedge_{1}^{R}\right) \frac{\Gamma \mapsto B_{1}, \Delta}{\Gamma \mapsto B_{1} \wedge B_{2}, \Delta} \\
& \left(\mapsto \wedge_{2}^{R}\right) \frac{\Gamma \mapsto B_{2}, \Delta}{\Gamma \mapsto B_{1} \wedge B_{2}, \Delta} \\
\left(\mapsto \vee_{1}^{L}\right) \frac{\Gamma, A_{1} \mapsto \Delta}{\Gamma, A_{1} \vee A_{2} \mapsto \Delta} & \left(\mapsto \vee^{R}\right) \frac{\Gamma \mapsto B_{1}, \Delta \Gamma \mapsto B_{2}, \Delta}{\Gamma \mapsto B_{1} \vee B_{2}, \Delta} \\
\left(\mapsto \vee_{2}^{L}\right) \frac{\Gamma, A_{2} \mapsto \Delta}{\Gamma, A_{1} \vee A_{2} \mapsto \Delta} &
\end{array}
$$

Definition 3.4 A sequent $\Gamma \mapsto \Delta$ is provable, denoted by $\vdash_{G_{1}} \Gamma \mapsto \Delta$ if there is a sequence $\left\{\Gamma_{1} \mapsto \Delta_{1}, \ldots, \Gamma_{n} \mapsto\right.$ Published by Sciedu Press
$\left.\Delta_{n}\right\}$ such that $\Gamma_{n} \mapsto \Delta_{n}=\Gamma \mapsto \Delta$, and for each $1 \leq i \leq n, \Gamma_{i} \mapsto \Delta_{i}$ is an axiom or is deduced from the previous sequents by one of the deduction rules.

Theorem 3.5 (The soundness and completeness theorem). For any sequent $\Gamma \mapsto \Delta$,

$$
\vdash_{G_{1}} \Gamma \mapsto \Delta \text { iff } \models_{G_{1}} \Gamma \mapsto \Delta
$$

4. The PROpositional Logic $G^{2}$

Definition 4.1 A sequent $\Gamma \Rightarrow \Delta$ is $G^{2}$-valid, denoted by $\models_{G^{2}} \Gamma \Rightarrow \Delta$ if for any assignment $v, v \models \Gamma$ implies $v \models \Delta$, where $v \models \Gamma$ if for every $A \in \Gamma, v(A)=1$; and $v \models \Delta$ if for each $B \in \Delta, v(B)=1$.

Proposition 4.2 Let $\Gamma, \Delta$ be sets of literals. $\models_{G^{2}} \Gamma \Rightarrow \Delta$ if and only if

$$
\Delta \subseteq \Gamma \text { or } \operatorname{incon}(\Gamma)
$$

Proof. Assume that $\Delta \subseteq \Gamma$ or $\operatorname{incon}(\Gamma)$. Then, $\models_{G^{2}} \Gamma \Rightarrow$ $\Delta$.

Conversely, assume that $\Delta \nsubseteq \Gamma$ and $\operatorname{con}(\Gamma)$. There is a literal $l \in \Delta-\Gamma$. Define an assignment $v$ such that for any propositional variable $p$,

$$
v(p)= \begin{cases}1 & \text { if } p \in \Gamma \\ 0 & \text { if } \neg p \in \Gamma \\ 0 & \text { if } p=l \\ 1 & \text { if } p=\neg l \\ 0 & \text { otherwise. }\end{cases}
$$

Then, $v \models \Gamma$ and $v \not \models \Delta$.

The Gentzen deduction system $G^{2}$ consists of the following axioms and deduction rules:

- Axioms:

$$
\left(A_{\Rightarrow}\right) \frac{\Delta \subseteq \Gamma \text { or } \operatorname{incon}(\Gamma)}{\Gamma \Rightarrow \Delta},
$$

where $\Delta, \Gamma$ are sets of literals.

- Deduction rules:

$$
\begin{array}{ll}
\left(\Rightarrow \wedge_{1}^{L}\right) \frac{\Gamma, A_{1} \Rightarrow \Delta}{\Gamma, A_{1} \wedge A_{2} \Rightarrow \Delta} & \left(\Rightarrow \wedge^{R}\right) \frac{\Gamma \Rightarrow B_{1}, \Delta \Gamma \Rightarrow B_{2}, \Delta}{\Gamma \Rightarrow B_{1} \wedge B_{2}, \Delta} \\
\left(\Rightarrow \wedge_{2}^{L}\right) \frac{\Gamma, A_{2} \Rightarrow \Delta}{\Gamma, A_{1} \wedge A_{2} \Rightarrow \Delta} & \\
\left(\Rightarrow \vee^{L}\right) \frac{\Gamma, A_{1} \Rightarrow \Delta \Gamma, A_{2} \Rightarrow \Delta}{\Gamma, A_{1} \vee A_{2} \Rightarrow \Delta} & \left(\Rightarrow \vee_{1}^{R}\right) \frac{\Gamma \Rightarrow B_{1}, \Delta}{\Gamma \Rightarrow B_{1} \vee B_{2}, \Delta} \\
& \left(\Rightarrow \vee_{2}^{R}\right) \frac{\Gamma \Rightarrow B_{2}, \Delta}{\Gamma \Rightarrow B_{1} \vee B_{2}, \Delta}
\end{array}
$$

Theorem 4.3 (The soundness theorem). For any sequent $\Gamma \Rightarrow \Delta$, if $\vdash_{G^{2}} \Gamma \Rightarrow \Delta$ then $\models_{G^{2}} \Gamma \Rightarrow \Delta$

Proof. We prove that each axiom is valid and each deduction rule preserves the validity. 
To verify the validity of the axiom, by Proposition 4.2, the axiom is valid.

To verify that $\left(\Rightarrow \wedge_{i}^{L}\right)$ preserves the validity, assume that for any assignment $v$,

$$
v \models \Gamma, A_{i} \text { implies } v \models \Delta
$$

For any assignment $v$, assume that $v \models \Gamma, A_{1} \wedge A_{2}$. Then, $v \models \Gamma, A_{i}$, and by the induction assumption, $v \models \Delta$.

To verify that $\left(\Rightarrow \wedge^{R}\right)$ preserves the validity, assume that for any assignment $v$,

$$
\begin{aligned}
& v \models \Gamma \text { implies } v \models B_{1}, \Delta \\
& v \models \Gamma \text { implies } v \models B_{2}, \Delta
\end{aligned}
$$

For any assignment $v$, assume that $v \models \Gamma$. By the induction assumption, $v \models B_{1}, \Delta$ and $v \models B_{2}, \Delta$. Hence, $v \models B_{1} \wedge B_{2}, \Delta$.

To verify that $\left(\Rightarrow \vee^{L}\right)$ preserves the validity, assume that for any assignment $v$,

$$
\begin{gathered}
v \models \Gamma, A_{1} \text { implies } v \models \Delta, \\
v \models \Gamma, A_{2} \text { implies } v \models \Delta
\end{gathered}
$$

For any assignment $v$, assume that $v \models \Gamma, A_{1} \vee A_{2}$. Then, either $v \models \Gamma, A_{1}$ or $v \models \Gamma, A_{2}$, and by the induction assumption, either case implies $v \models \Delta$.

To verify that $\left(\Rightarrow \vee_{i}^{R}\right)$ preserves the validity, assume that for any assignment $v$,

$$
v \models \Gamma \text { implies } v \models B_{i}, \Delta
$$

For any assignment $v$, assume that $v \models \Gamma$. By the induction assumption, $v \models B_{i}, \Delta$. Hence, $v \models B_{1} \vee B_{2}, \Delta$.

\subsection{The completeness theorem of $G^{2}$}

Theorem 4.4 (The completeness theorem). For any sequent $\Gamma \Rightarrow \Delta$, if $\models_{G^{2}} \Gamma \Rightarrow \Delta$ then $\vdash_{G^{2}} \Gamma \Rightarrow \Delta$.

Proof. Given a sequent $\Gamma \Rightarrow \Delta$, we construct a tree $T$ as follows:

- the root of $T$ is $\Gamma \Rightarrow \Delta$;

- if for each sequent $\Gamma^{\prime} \Rightarrow \Delta^{\prime}$ at a node, $\Gamma^{\prime}, \Delta^{\prime}$ are sets of literals then the node is a leaf; and

- if a sequent $\Gamma^{\prime} \Rightarrow \Delta^{\prime}$ at a nonleaf node of $T$ is not an axiom then the node has the direct child nodes

$$
\left\{\begin{array}{l}
{\left[\begin{array}{c}
\Gamma_{1}, A_{1} \Rightarrow \Delta_{1} \\
\Gamma_{1}, A_{2} \Rightarrow \Delta_{1}
\end{array} \quad \text { if } \Gamma^{\prime} \Rightarrow \Delta^{\prime}=\Gamma_{1}, A_{1} \wedge A_{2} \Rightarrow \Delta_{1}\right.} \\
\left\{\begin{array}{l}
\Gamma_{1} \Rightarrow B_{1}, \Delta_{1} \\
\Gamma_{1} \Rightarrow B_{2}, \Delta_{1} \\
\Gamma_{1}, A_{1} \Rightarrow \Delta_{1} \\
\Gamma_{1}, A_{2} \Rightarrow \Delta_{1}
\end{array} \text { if } \Gamma^{\prime} \Rightarrow \Delta^{\prime}=\Gamma_{1} \Rightarrow B_{1} \wedge B_{2}, \Delta_{1}\right.
\end{array}\right.
$$

where $\left[\begin{array}{l}\delta_{1} \\ \delta_{2}\end{array}\right.$ represents that $\delta_{1}, \delta_{2}$ are at a same child node; and $\left\{\begin{array}{l}\delta_{1} \\ \delta_{2}\end{array}\right.$ represents that $\delta_{1}, \delta_{2}$ are at different direct child nodes;

Theorem 4.5 If there is a branch $\xi \subseteq T$ such that the leaf of $\xi$ is not an axiom in $G^{2}$ then there is an assignment $v$ such that $v \forall \forall_{G^{2}} \Gamma \Rightarrow \Delta$.

Proof. Assume that the leaf of $\xi$ is not an axiom in $G^{2}$, and let the leaf be $\Gamma^{\prime} \Rightarrow \Delta^{\prime}$, Then, By Proposition 4.2, there is an assignment $v$ such that $v \not \forall_{G^{2}} \Gamma^{\prime} \Rightarrow \Delta^{\prime}$.

We shall prove that for each node $\Gamma_{1} \Rightarrow \Delta_{1}$ of $\xi, v \not \forall_{G^{2}}$ $\Gamma_{1} \Rightarrow \Delta_{1}$. There are the following cases for $\Gamma_{1} \Rightarrow \Delta_{1}$.

Case 1. $\Gamma_{1} \Rightarrow \Delta_{1}=\Gamma_{2}, A_{1}, A_{2} \Rightarrow \Delta_{2} \in \xi$ is a direct child nodes of $\Gamma_{2}, A_{1} \wedge A_{2} \Rightarrow \Delta_{2} \in \xi$. By the induction assumption,

$$
v \not \forall_{G^{2}} \Gamma_{2}, A_{1}, A_{2} \mapsto \Delta_{2}
$$

and we have that $v \not \forall_{G^{2}} \Gamma_{2}, A_{1} \wedge A_{2} \mapsto \Delta_{2}$.

Case 2. $\Gamma_{1} \Rightarrow \Delta_{1}=\Gamma_{2} \Rightarrow B_{i}, \Delta_{2} \in \xi$ is a direct child node of $\Gamma_{2} \Rightarrow B_{1} \wedge B_{2}, \Delta_{2} \in \xi$. By the induction assumption,

$$
v \not \forall_{G^{2}} \Gamma_{2} \Rightarrow B_{i}, \Delta_{2}
$$

and we have that $v \not \forall_{G^{2}} \Gamma_{2} \Rightarrow B_{1} \wedge B_{2}, \Delta_{2}$.

Case 3. $\Gamma_{1} \Rightarrow \Delta_{1}=\Gamma_{2}, A_{i} \Rightarrow \Delta_{2} \in \xi$ is a direct child node of $\Gamma_{2}, A_{1} \vee A_{2} \Rightarrow \Delta_{2} \in \xi$. By the induction assumption,

$$
v \not \forall_{G^{2}} \Gamma_{2}, A_{i} \Rightarrow \Delta_{2}
$$

and we have that $v \forall_{G^{2}} \Gamma_{2}, A_{1} \vee A_{2} \Rightarrow \Delta_{2}$.

Case 4. $\Gamma_{1} \Rightarrow \Delta_{1}=\Gamma_{2} \Rightarrow B_{1}, B_{2}, \Delta_{2} \in \xi$ is a direct child node of $\Gamma_{2} \Rightarrow B_{1} \vee B_{2}, \Delta_{2} \in \xi$. By the induction assumption,

$$
v \not \forall_{G^{2}} \Gamma_{2} \Rightarrow B_{1}, B_{2}, \Delta_{2}
$$

that is,

$$
v \forall G_{G^{2}} \Gamma_{2} \Rightarrow B_{1}, \Delta_{2}
$$




$$
v \not \forall_{G^{2}} \Gamma_{2} \Rightarrow B_{2}, \Delta_{2}
$$

and we have that $v \not G_{G^{2}} \Gamma_{2} \Rightarrow B_{1} \vee B_{2} \Delta_{2}$.

Theorem 4.6 If each leaf $\Gamma^{\prime} \Rightarrow \Delta^{\prime}$ of $T$ is an axiom in $G^{2}$ then $T$ is a proof tree of $\Gamma \Rightarrow \Delta$ in $G^{2}$.

Proof. The theorem follows directly from the definition of $T$.

\subsection{The propositional logic $G_{2}$}

Definition 4.7 A sequent $\Gamma \mapsto \Delta$ is $G_{2}$-valid, denoted by $\models_{G_{2}} \Gamma \mapsto \Delta$ if there is an assignment $v$ such that $v \models \Gamma$ and $v \models \Delta$, where $v \models \Gamma$ if for every $A \in \Gamma, v(A)=1$; and $v \models \Delta$ if for some $B \in \Delta, v(B)=0$.

Proposition 4.8 Let $\Gamma, \Delta$ be sets of literals. $\models_{G_{2}} \Gamma \mapsto \Delta$ if and only if

$$
\Delta \nsubseteq \Gamma \& \operatorname{con}(\Gamma)
$$

The Gentzen deduction system $G_{2}$ consists of the following axioms and deduction rules:

- Axioms:

$$
\left(A_{\mapsto}\right) \frac{\Delta \nsubseteq \Gamma \& \operatorname{con}(\Gamma)}{\Gamma \mapsto \Delta},
$$

where $\Delta, \Gamma$ are sets of literals.

- Deduction rules:

$$
\begin{array}{ll}
\left(\mapsto \wedge^{L}\right) \frac{\Gamma, A_{1} \mapsto \Delta \Gamma, A_{2} \mapsto \Delta}{\Gamma, A_{1} \wedge A_{2} \mapsto \Delta} & \left(\mapsto \wedge_{1}^{R}\right) \frac{\Gamma \mapsto B_{1}, \Delta}{\Gamma \mapsto B_{1} \wedge B_{2}, \Delta} \\
& \left(\mapsto \wedge_{2}^{R}\right) \frac{\Gamma \mapsto B_{2}, \Delta}{\Gamma \mapsto B_{1} \wedge B_{2}, \Delta} \\
\left(\mapsto \vee_{1}^{L}\right) \frac{\Gamma, A_{1} \mapsto \Delta}{\Gamma, A_{1} \vee A_{2} \mapsto \Delta} & \left(\mapsto \vee^{R}\right) \frac{\Gamma \mapsto B_{1}, \Delta \Gamma \mapsto B_{2}, \Delta}{\Gamma \mapsto B_{1} \vee B_{2}, \Delta} \\
\left(\mapsto \vee_{2}^{L}\right) \frac{\Gamma, A_{2} \mapsto \Delta}{\Gamma, A_{1} \vee A_{2} \mapsto \Delta} &
\end{array}
$$

Theorem 4.9 (The soundness theorem). For any sequent $\Gamma \mapsto \Delta$, if $\vdash_{G_{2}} \Gamma \mapsto \Delta$ then $\models_{G_{2}} \Gamma \mapsto \Delta$.

Proof. We prove that each axiom is valid and each deduction rule preserves the validity.

To verify the validity of the axiom, by Proposition 4.8, the axiom is valid.

To verify that $\left(\mapsto \wedge^{L}\right)$ preserves the validity, assume that there is an assignment $v$ such that

$$
\begin{aligned}
& v \models \Gamma, A_{1} \& v \models \Delta, \\
& v \models \Gamma, A_{2} \& v \models \Delta .
\end{aligned}
$$

For this assignment $v, v \models \Gamma, A_{1} \wedge A_{2}$ and $v \models \Delta$.

To verify that $\left(\mapsto \wedge_{i}^{R}\right)$ preserves the validity, assume that there is an assignment $v$ such that

$$
v \models \Gamma \& v \models B_{i}, \Delta .
$$

For this assignment $v, v \models \Gamma$ and $v \models B_{1} \wedge B_{2}, \Delta$.

Published by Sciedu Press
To verify that $\left(\mapsto \vee_{i}^{L}\right)$ preserves the validity, assume that there is an assignment $v$ such that

$$
v \models \Gamma, A_{i} \& v \models \Delta .
$$

For this assignment $v, v \models \Gamma, A_{1} \vee A_{2}$ and $v \models \Delta$.

To verify that $\left(\mapsto \vee^{R}\right)$ preserves the validity, assume that there is an assignment $v$ such that

$$
\begin{aligned}
& v \models \Gamma \& v \models B_{1}, \Delta, \\
& v \models \Gamma \& v \models B_{2}, \Delta .
\end{aligned}
$$

For this assignment $v, v \models \Gamma$ and $v \models B_{1} \vee B_{2}, \Delta$.

\subsection{The completeness theorem of $G_{2}$}

Theorem 4.10 (The completeness theorem). For any sequent $\Gamma \mapsto \Delta$, if $\models_{G_{2}} \Gamma \mapsto \Delta$ then $\vdash_{G_{2}} \Gamma \mapsto \Delta$.

Proof. Given a sequent $\Gamma \mapsto \Delta$, we construct a tree $T$ as follows:

- the root of $T$ is $\Gamma \mapsto \Delta$;

- if for each sequent $\Gamma^{\prime} \mapsto \Delta^{\prime}$ at a node, $\Gamma^{\prime}, \Delta^{\prime}$ are sets of literals then the node is a leaf; and

- if a sequent $\Gamma^{\prime} \mapsto \Delta^{\prime}$ at a nonleaf node of $T$ is not an axiom then the node has the direct child nodes

$\left\{\begin{array}{l}{\left[\begin{array}{c}\Gamma_{1}, A_{1} \mapsto \Delta_{1} \\ \Gamma_{1}, A_{2} \mapsto \Delta_{1}\end{array} \quad \text { if } \Gamma^{\prime} \mapsto \Delta^{\prime}=\Gamma_{1}, A_{1} \wedge A_{2} \mapsto \Delta_{1}\right.} \\ \left\{\begin{array}{l}\Gamma_{1} \mapsto B_{1}, \Delta_{1} \\ \Gamma_{1} \mapsto B_{2}, \Delta_{1} \\ \Gamma_{1}, A_{1} \mapsto \Delta_{1} \\ \Gamma_{1}, A_{2} \mapsto \Delta_{1}\end{array} \quad \text { if } \Gamma^{\prime} \mapsto \Delta^{\prime}=\Gamma_{1} \mapsto B_{1} \wedge B_{2}, \Delta_{1}\right.\end{array}\right.$

Theorem 4.11 If there is a branch $\xi \subseteq T$ such that the leaf of $\xi$ is a precondition of the axiom in $G_{2}$ then $\vdash_{G_{2}} \Gamma \mapsto \Delta$.

Proof. Assume that the leaf of $\xi$ is a precondition of the axiom in $G_{2}$, by $(\mapsto A), \vdash_{G_{2}} \Gamma^{\prime} \mapsto \Delta^{\prime}$.

We shall prove that for each node $\Gamma_{1} \mapsto \Delta_{1}$ of $\xi, \vdash_{G_{2}} \Gamma_{1} \mapsto$ $\Delta_{1}$. There are the following cases for $\Gamma_{1} \mapsto \Delta_{1}$.

Case 1. $\Gamma_{1} \mapsto \Delta_{1}=\Gamma_{2}, A_{1}, A_{2} \mapsto \Delta_{2} \in \xi$ is a direct child node of $\Gamma_{2}, A_{1} \wedge A_{2} \mapsto \Delta_{2} \in \xi$. By the induction assumption,

$$
\begin{aligned}
& \vdash_{G_{2}} \Gamma_{2}, A_{1} \mapsto \Delta_{2}, \\
& \vdash_{G_{2}} \Gamma_{2}, A_{2} \mapsto \Delta_{2},
\end{aligned}
$$

and by $\left(\mapsto \wedge^{L}\right)$, we have that $\vdash_{G_{2}} \Gamma_{2}, A_{1} \wedge A_{2} \mapsto \Delta_{2}$.

Case 2. $\Gamma_{1} \mapsto \Delta_{1}=\Gamma_{2} \mapsto B_{i}, \Delta_{2} \in \xi$ is a direct child node of $\Gamma_{2} \mapsto B_{1} \wedge B_{2}, \Delta_{2} \in \xi$. By the induction assump- 
tion,

$$
\vdash_{G_{2}} \Gamma_{2} \mapsto B_{i}, \Delta_{2},
$$

and by $\left(\mapsto \wedge_{i}^{R}\right)$, we have that $\vdash_{G_{2}} \Gamma_{2} \mapsto B_{1} \wedge B_{2}, \Delta_{2}$.

Case 3. $\Gamma_{1} \mapsto \Delta_{1}=\Gamma_{2}, A_{i} \mapsto \Delta_{2} \in \xi$ is a direct child node of $\Gamma_{2}, A_{1} \vee A_{2} \mapsto \Delta_{2} \in \xi$. By the induction assumption,

$$
\vdash_{G_{2}} \Gamma_{2}, A_{i} \mapsto \Delta_{2},
$$

and by $\left(\mapsto \vee_{i}^{L}\right)$, we have that $\vdash_{G_{2}} \Gamma_{2}, A_{1} \vee A_{2} \mapsto \Delta_{2}$.

Case 4. $\Gamma_{1} \mapsto \Delta_{1}=\Gamma_{2} \mapsto B_{1}, B_{2}, \Delta_{2} \in \xi$ is a direct child node of $\Gamma_{2} \mapsto B_{1} \vee B_{2}, \Delta_{2} \in \xi$. By the induction assumption,

$$
\vdash_{G_{2}} \Gamma_{2} \mapsto B_{1}, B_{2}, \Delta_{2}
$$

that is,

$$
\begin{aligned}
& \vdash_{G_{2}} \Gamma_{2} \mapsto B_{1}, \Delta_{2}, \\
& \vdash_{G_{2}} \Gamma_{2} \mapsto B_{2}, \Delta_{2},
\end{aligned}
$$

and by $\left(\mapsto \vee^{R}\right)$, we have that $\vdash_{G_{2}} \Gamma_{2} \mapsto B_{1} \vee B_{2} \Delta_{2}$

Theorem 4.12 If each leaf $\Gamma^{\prime} \mapsto \Delta^{\prime}$ of $T$ is not an axiom in $G_{2}$ then $T$ is a proof tree of $\Gamma \Rightarrow \Delta$ in $G^{2}$.

Proof. The theorem follows directly from the definition of $T$.

\section{THE PROPOSITIONAL LOGIC $G^{3}$}

Given a sequent $\Gamma \Rightarrow \Delta$, we say that $v$ satisfies $\Gamma \Rightarrow \Delta$, denoted by $v \models_{G^{3}} \Gamma \Rightarrow \Delta$, if 1) that for each formula $A \in \Gamma, v(A)=1$ implies 2) that for some formula $A \in \Delta, v(A)=0$.

A sequent $\Gamma \Rightarrow \Delta$ is valid, denoted by $\models_{G^{3}} \Gamma \Rightarrow \Delta$, if for any assignment $v, v \models_{G^{3}} \Gamma \Rightarrow \Delta$.

Proposition 5.1 Let $\Gamma, \Delta$ be sets of literals. $\models_{G^{3}} \Gamma \Rightarrow \Delta$ if and only if

$$
\operatorname{incon}(\Gamma) \text { or } \operatorname{incon}(\neg \Delta) \text { or } \Gamma \cap \neg \Delta \neq \emptyset .
$$

Proof. $\models_{G^{3}} \Gamma \Rightarrow \Delta$ iff for any assignment $v, 1$ ) that for each formula $A \in \Gamma, v(A)=1$ implies 2) that for some formula $A \in \Delta, v(A)=0$; iff for any assignment $v, 1$ ) that for each formula $A \in \Gamma, v(A)=1$ implies 2) that for some formula $A \in \Delta, v(\neg A)=1$; iff $\models_{G_{1}} \Gamma \Rightarrow \neg \Delta$, iff

$$
\operatorname{incon}(\Gamma) \text { or } \operatorname{incon}(\neg \Delta) \text { or } \Gamma \cap \neg \Delta \neq \emptyset \text {, }
$$

where $\neg \Delta=\{\neg B: B \in \Delta\}$.

Proposition 5.2 Let $\Gamma, \Delta$ be sets of literals. $\operatorname{incon}(\Gamma)$ or $\operatorname{incon}(\neg \Delta)$ or $\Gamma \cap \neg \Delta \neq \emptyset$ if and only if $\operatorname{incon}(\Gamma \cup \Delta)$.

The Gentzen deduction system $G^{3}$ contains the following axioms and deduction rules:

- Axioms:

$$
\frac{i n \operatorname{con}(\Gamma) \text { or } i n \operatorname{con}(\neg \Delta) \text { or } \Gamma \cap \neg \Delta \neq \emptyset}{\Gamma \Rightarrow \Delta},
$$

where $\Gamma, \Delta$ are sets of literals.

- The deduction rules for connectives:

$$
\begin{array}{ll}
\left(\Rightarrow \wedge_{1}^{L}\right) \frac{\Gamma, A_{1} \Rightarrow \Delta}{\Gamma, A_{1} \wedge A_{2} \Rightarrow \Delta} & \left(\Rightarrow \wedge_{1}^{R}\right) \frac{\Gamma \Rightarrow B_{1}, \Delta}{\Gamma \Rightarrow B_{1} \wedge B_{2}, \Delta} \\
\left(\Rightarrow \wedge_{2}^{L}\right) \frac{\Gamma, A_{2} \Rightarrow \Delta}{\Gamma, A_{1} \wedge A_{2} \Rightarrow \Delta} & \left(\Rightarrow \wedge_{2}^{R}\right) \frac{\Gamma \Rightarrow B_{2}, \Delta}{\Gamma \Rightarrow B_{1} \wedge B_{2}, \Delta} \\
\left(\Rightarrow \vee^{L}\right) \frac{\Gamma, A_{1} \Rightarrow \Delta \Gamma, A_{2} \Rightarrow \Delta}{\Gamma, A_{1} \vee A_{2} \Rightarrow \Delta} & \left(\Rightarrow \vee^{R}\right) \frac{\Gamma \Rightarrow B_{1}, \Delta \Gamma \Rightarrow B_{2}, \Delta}{\Gamma \Rightarrow B_{1} \vee B_{2}, \Delta}
\end{array}
$$

Definition $5.3 \vdash_{G^{3}} \Gamma \Rightarrow \Delta$ if there is a sequence $\left\{\Gamma_{1} \Rightarrow\right.$ $\Delta_{1}, \ldots, \Gamma_{n} \Rightarrow \Delta_{n}$ such that $\Gamma_{n} \Rightarrow \Delta_{n}=\Gamma \Rightarrow \Delta$, and for each $1 \leq i \leq n, \Gamma_{i} \Rightarrow \Delta_{i}$ is an axiom or is deduced from the previous sequents by one of the deduction rules in $G^{3}$.

Theorem 5.4 (The soundness theorem). For any sequent $\Gamma \Rightarrow \Delta$, if $\vdash_{G^{3}} \Gamma \Rightarrow \Delta$ then $\models_{G^{3}} \Gamma \Rightarrow \Delta$.

Proof. We prove that each axiom is valid and each deduction rule preserves the validity.

To verify the validity of the axiom, by Proposition 5.1, the axiom is valid.

To verify that $\left(\Rightarrow \wedge^{R_{1}}\right)$ preserves the validity, assume that for any assignment $v$,

$$
v \models \Gamma, A_{1} \text { implies } v \models \Delta \text {. }
$$

For any assignment $v$, assume that $v \models \Gamma, A_{1} \wedge A_{2}$. Then, $v \models \Gamma, A_{1}$. By the induction assumption, $v \models \Delta$.

To verify that $\left(\Rightarrow \wedge^{R}\right)$ preserves the validity, assume that for any assignment $v$

$$
v \models \Gamma \text { implies } v \models B_{1}, \Delta \text {. }
$$

For any assignment $v$, assume that $v \models \Gamma$. By the induction assumption, $v \models B_{1}, \Delta$. If $v \models \Delta$ then $v \models B_{1} \wedge B_{2}, \Delta$; otherwise, $v \models B_{1}$ and so $v \models B_{1} \wedge B_{2}$, i.e., $v \models B_{1} \wedge B_{2}, \Delta$.

To verify that $\left(\vee^{L}\right)$ preserves the validity, assume that for any assignment $v$,

$$
\begin{gathered}
v \models \Gamma, A_{1} \text { implies } v \models \Delta \\
v \models \Gamma, A_{2} \text { implies } v \models \Delta .
\end{gathered}
$$

For any assignment $v$, assume that $v \models \Gamma, A_{1} \vee A_{2}$. If $v \models A_{1}$ then $v \models \Gamma, A_{1}$, and by the induction assumption, $v \models \Delta$; and if $v \models A_{2}$ then, $v \models \Gamma, A_{1}$, and by the induction assumption, $v \models \Delta$.

To verify that $\left(\Rightarrow \vee^{R}\right)$ preserves the validity, assume that for any assignment $v$,

$$
v \models \Gamma \text { implies } v \models B_{1}, \Delta
$$

ISSN 1927-6974 E-ISSN 1927-6982 


$$
v \models \Gamma \text { implies } v \models B_{2}, \Delta .
$$

For any assignment $v$, assume that $v \models \Gamma$. By the induction assumption, $v \models B_{1}, \Delta$ and $v \models B_{1}, \Delta$. If $v \models \Delta$ then $v \models B_{1} \vee B_{2}, \Delta$; otherwise, $v \models B_{1} ; v \models B_{2}$, and so $v \models B_{1} \vee B_{2}, \Delta$.

\subsection{The completeness theorem of $G^{3}$}

Theorem 5.5 (The completeness theorem). For any sequent $\Gamma \Rightarrow \Delta$, if $\models_{G^{3}} \Gamma \Rightarrow \Delta$ then $\vdash_{G^{3}} \Gamma \Rightarrow \Delta$.

Proof. Given a sequent $\Gamma \Rightarrow \Delta$, we construct a tree $T$ as follows:

- the root of $T$ is $\Gamma \Rightarrow \Delta$;

- if for each sequent $\Gamma^{\prime} \Rightarrow \Delta^{\prime}$ at a node, $\Gamma^{\prime}, \Delta^{\prime}$ are sets of literals then the node is a leaf; and

- if a sequent $\Gamma^{\prime} \Rightarrow \Delta^{\prime}$ at a nonleaf node of $T$ is not an axiom then the node has the direct child nodes

$$
\left\{\begin{array}{l}
{\left[\begin{array}{l}
\Gamma_{1}, A_{1} \Rightarrow \Delta_{1} \\
\Gamma_{1}, A_{2} \Rightarrow \Delta_{1} \\
\Gamma_{1} \Rightarrow B_{1}, \Delta_{1} \\
\Gamma_{1} \Rightarrow B_{2}, \Delta_{1}
\end{array} \quad \text { if } \Gamma^{\prime} \Rightarrow \Delta^{\prime}=\Gamma_{1}, A_{1} \wedge A_{2} \Rightarrow \Delta_{1}=\Gamma_{1} \Rightarrow B_{1} \wedge B_{2}, \Delta_{1}\right.} \\
\left\{\begin{array}{l}
\Gamma_{1}, A_{1} \Rightarrow \Delta_{1} \\
\Gamma_{1}, A_{2} \Rightarrow \Delta_{1} \\
\Gamma_{1} \Rightarrow B_{1}, \Delta_{1} \\
\Gamma_{1} \Rightarrow B_{2}, \Delta_{1}
\end{array} \quad \text { if } \Gamma^{\prime} \Rightarrow \Delta^{\prime}=\Gamma_{1}, A_{1} \vee A_{2} \Rightarrow \Delta_{1}\right.
\end{array}\right.
$$

Theorem 5.6 If there is a branch $\xi \subseteq T$ such that the leaf of $\xi$ is not an axiom in $G^{3}$ then there is an assignment $v$ such that $v \forall \forall_{G^{3}} \Gamma \Rightarrow \Delta$.

Proof. Assume that the leaf of $\xi$ is not an axiom in $G^{3}$, and let the leaf be $\Gamma^{\prime} \Rightarrow \Delta^{\prime}$, Then, By Proposition 5.1, there is an assignment $v$ such that $v \forall_{G^{3}} \Gamma^{\prime} \Rightarrow \Delta^{\prime}$.

We shall prove that for each node $\Gamma_{1} \Rightarrow \Delta_{1}$ of $\xi, v \not \forall_{G^{3}}$ $\Gamma_{1} \Rightarrow \Delta_{1}$. There are the following cases for $\Gamma_{1} \Rightarrow \Delta_{1}$.

Case 1. $\Gamma_{1} \Rightarrow \Delta_{1}=\Gamma_{2}, A_{1}, A_{2} \Rightarrow \Delta_{2} \in \xi$ is a direct child node of $\Gamma_{2}, A_{1} \wedge A_{2} \Rightarrow \Delta_{2} \in \xi$. By the induction assumption,

$$
v \not \forall_{G^{3}} \Gamma_{2}, A_{1}, A_{2} \Rightarrow \Delta_{2},
$$

and we have that $v \not \forall_{G^{3}} \Gamma_{2}, A_{1} \wedge A_{2} \Rightarrow \Delta_{2}$.

Case 2. $\Gamma_{1} \Rightarrow \Delta_{1}=\Gamma_{2} \Rightarrow B_{1}, B_{2}, \Delta_{2} \in \xi$ is a direct child node of $\Gamma_{2} \Rightarrow B_{1} \wedge B_{2}, \Delta_{2} \in \xi$. By the induction assumption,

$$
v \not \forall_{G^{3}} \Gamma_{2} \Rightarrow B_{1}, B_{2}, \Delta_{2},
$$

and we have that $v \not \forall_{G^{3}} \Gamma_{2} \Rightarrow B_{1} \wedge B_{2}, \Delta_{2}$.

Case 3. $\Gamma_{1} \Rightarrow \Delta_{1}=\Gamma_{2}, A_{i} \Rightarrow \Delta_{2} \in \xi$ is a direct child node of $\Gamma_{2}, A_{1} \vee A_{2} \Rightarrow \Delta_{2} \in \xi$. By the induction assumpPublished by Sciedu Press tion,

$$
v \not \forall_{G^{3}} \Gamma_{2}, A_{i} \Rightarrow \Delta_{2},
$$

and we have that $v \not \forall_{G^{3}} \Gamma_{2}, A_{1} \vee A_{2} \Rightarrow \Delta_{2}$.

Case 4. $\Gamma_{1} \Rightarrow \Delta_{1}=\Gamma_{2} \Rightarrow B_{i}, \Delta_{2} \in \xi$ is a direct child node of $\Gamma_{2} \Rightarrow B_{1} \vee B_{2}, \Delta_{2} \in \xi$. By the induction assumption,

$$
v \not \forall_{G^{3}} \Gamma_{2} \Rightarrow B_{i}, \Delta_{2},
$$

and we have that $v \not \forall_{G^{3}} \Gamma_{2} \Rightarrow B_{1} \vee B_{2} \Delta_{2}$.

Theorem 5.7 If each leaf $\Gamma^{\prime} \Rightarrow \Delta^{\prime}$ of $T$ is an axiom in $G^{3}$ then $T$ is a proof tree of $\Gamma \Rightarrow \Delta$ in $G^{3}$.

Proof. The theorem follows directly from the definition of $T$.

\subsection{The propositional logic $G_{3}$}

Definition 5.8 A sequent $\Gamma \mapsto \Delta$ is valid in $G_{3}$, denoted by $\models_{G_{3}} \Gamma \mapsto \Delta$ if there is an assignment $v$ such that $v \models \Gamma$ and $v \models \Delta$, where $v \models \Gamma$ if for each $A \in \Gamma, v(A)=1$; and $v \models \Delta$ if for each $B \in \Delta, v(B)=1$.

A sequent $\Gamma \mapsto \Delta$ is not valid in $G_{3}$ if $\Gamma \mapsto \Delta$ is unsatisfiable in $G_{3}$, i.e., there is no assignment $v$ such that $v \models \Gamma$ and $v \models \Delta$, equivalently, for any assignment $v, v \models \Gamma$ implies $v \models \Delta$.

Lemma 5.9 Given two sets $\Gamma, \Delta$ of literals, $\models_{G_{3}} \Gamma \mapsto \Delta$ if and only if $\Gamma$ and $\neg \Delta$ are consistent, and $\Gamma \cap \neg \Delta=\emptyset$, equivalently, $\operatorname{con}(\Gamma \cup \Delta)$.

The Gentzen deduction system $G_{3}$ consists of the following axioms and deduction rules:

- Axioms:

$$
\left(A^{\prime} \rightarrow\right) \frac{\operatorname{con}(\Gamma) \& \operatorname{con}(\neg \Delta) \& \Gamma \cap \neg \Delta=\emptyset}{\Gamma \mapsto \Delta},
$$

where $\Delta, \Gamma$ are sets of literals.

- Deduction rules:

$$
\begin{array}{lll}
\left(\mapsto \wedge^{L}\right) \frac{\Gamma, A_{1} \mapsto \Delta \Gamma, A_{2} \mapsto \Delta}{\Gamma, A_{1} \wedge A_{2} \mapsto \Delta} & \left(\mapsto \wedge^{R}\right) \frac{\Gamma \mapsto B_{1}, \Delta \Gamma \mapsto B_{2}, \Delta}{\Gamma \mapsto B_{1} \wedge B_{2}, \Delta} \\
\left(\mapsto \vee_{1}^{L}\right) \frac{\Gamma, A_{1} \mapsto \Delta}{\Gamma, A_{1} \vee A_{2} \mapsto \Delta} & \left(\mapsto \vee_{1}^{R}\right) \frac{\Gamma \mapsto B_{1}, \Delta}{\Gamma \mapsto B_{1} \vee B_{2}, \Delta} \\
\left(\mapsto \vee_{2}^{L}\right) \frac{\Gamma, A_{2} \mapsto \Delta}{\Gamma, A_{1} \vee A_{2} \mapsto \Delta} & \left(\mapsto \vee_{2}^{R}\right) \frac{\Gamma \mapsto B_{2}, \Delta}{\Gamma \mapsto B_{1} \vee B_{2}, \Delta}
\end{array}
$$

Definition 5.10 A sequent $\Gamma \mapsto \Delta$ is provable, denoted by $\vdash_{G_{3}} \Gamma \mapsto \Delta$ if there is a sequence $\left\{\Gamma_{1} \mapsto \Delta_{1}, \ldots, \Gamma_{n} \mapsto\right.$ $\left.\Delta_{n}\right\}$ such that $\Gamma_{n} \mapsto \Delta_{n}=\Gamma \mapsto \Delta$, and for each $1 \leq i \leq n, \Gamma_{i} \mapsto \Delta_{i}$ is an axiom or is deduced from the previous sequents by one of the deduction rules.

\subsection{The soundness and completeness theorem of $G_{3}$}

Theorem 5.11 (The soundness theorem). For any sequent $\Gamma \mapsto \Delta$,

$$
\vdash_{G_{3}} \Gamma \mapsto \Delta \text { implies } \models G_{3} \Gamma \mapsto \Delta \text {. }
$$


Proof. We prove that each axiom is valid and each deduction rule preserves the validity.

To verify the validity of the axiom, assume that $\operatorname{con}(\Gamma)$, $\operatorname{con}(\neg \Delta)$ and $\Gamma \cap \neg \Delta=\emptyset$. By Lemma 5.2.8, there is an assignment $v$ such that $v \models_{G_{3}} \Gamma \mapsto \Delta$.

To verify that $\left(\mapsto \wedge^{L}\right)$ preserves the validity, assume that there is an assignment $v$ such that

$$
\begin{aligned}
& v\left(\Gamma, A_{1}\right)=1 \& v \models \Delta, \\
& v\left(\Gamma, A_{2}\right)=1 \& v \models \Delta .
\end{aligned}
$$

For this very assignment $v, v\left(\Gamma, A_{1} \wedge A_{2}\right)=1$ and $v \models \Delta$. To verify that $\left(\mapsto \wedge^{R}\right)$ preserves the validity, assume that there is an assignment $v$ such that

$$
\begin{gathered}
v \models \Gamma \& v\left(\Delta, B_{1}\right)=1, \\
v \models \Gamma \& v\left(\Delta, B_{2}\right)=1 .
\end{gathered}
$$

For this very assignment $v, v \models \Gamma$ and $v\left(\Delta, B_{1} \wedge B_{2}\right)=1$. To verify that $\left(\mapsto \vee_{1}^{L}\right)$ preserves the validity, assume that there is an assignment $v$ such that $v\left(\Gamma, A_{1}\right)=1$ and $v \models \Delta$. For this very assignment $v, v\left(\Gamma, A_{1} \vee A_{2}\right)=1$ and $v \models \Delta$.

To verify that $\left(\mapsto \vee_{1}^{R}\right)$ preserves the validity, assume that there is an assignment $v$ such that $v \models \Gamma$ and $v\left(\Delta, B_{1}\right)=1$. For this very assignment $v, v \models \Gamma$ and $v\left(\Delta, B_{1} \vee B_{2}\right)=1$.

Theorem 5.12 (The completeness theorem). For any sequent $\Gamma \mapsto \Delta$,

$$
\models_{G_{3}} \Gamma \mapsto \Delta \text { implies } \vdash_{G_{3}} \Gamma \mapsto \Delta \text {. }
$$

Proof. Given a sequent $\Gamma \mapsto \Delta$, we construct a tree $T$ as follows:

- the root of $T$ is $\Gamma \mapsto \Delta$;

- if for each sequent $\Gamma^{\prime} \mapsto \Delta^{\prime}$ at a node, $\Gamma^{\prime}, \Delta^{\prime}$ are sets of literals then the node is a leaf; and

- if a sequent $\Gamma^{\prime} \mapsto \Delta^{\prime}$ at a nonleaf node of $T$ is not an axiom then the node has the direct child nodes

$$
\left\{\begin{array}{l}
{\left[\begin{array}{c}
\Gamma_{1}, A_{1} \mapsto \Delta_{1} \\
\Gamma_{1}, A_{2} \mapsto \Delta_{1} \\
\Gamma_{1} \mapsto B_{1}, \Delta_{1} \\
\Gamma_{1} \mapsto B_{2}, \Delta_{1}
\end{array} \quad \text { if } \Gamma^{\prime} \mapsto \Delta^{\prime} \mapsto \Delta^{\prime}=\Gamma_{1} \mapsto A_{1} \wedge B_{2}, \Delta_{1} \mapsto \Delta_{1}\right.} \\
\left\{\begin{array}{l}
\Gamma_{1}, A_{1} \mapsto \Delta_{1} \\
\Gamma_{1}, A_{2} \mapsto \Delta_{1} \\
\Gamma_{1} \mapsto B_{1}, \Delta_{1} \\
\Gamma_{1} \mapsto B_{2}, \Delta_{1}
\end{array} \quad \text { if } \Gamma^{\prime} \mapsto \Delta^{\prime}=\Gamma_{1}, A_{1} \vee A_{2} \mapsto \Delta_{1}\right.
\end{array}\right.
$$

Theorem 5.13 If there is a branch $\xi \subseteq T$ such that the leaf of $\xi$ is an axiom in $G_{3}$ then $\vdash_{G_{3}} \Gamma \mapsto \Delta$.
Proof. Assume that the leaf of $\xi$ is an axiom in $G_{3}$. Let the leaf be $\Gamma^{\prime} \mapsto \Delta^{\prime}$. Then, $\operatorname{con}\left(\Gamma^{\prime}\right), \operatorname{con}\left(\neg \Delta^{\prime}\right)$ and $\Gamma^{\prime} \cap \neg \Delta^{\prime}=\emptyset$, and by $(A), \vdash_{G_{3}} \Gamma^{\prime} \Rightarrow \Delta^{\prime}$.

We shall prove that for each node $\Gamma_{1} \mapsto \Delta_{1}$ of $\xi, \vdash_{G_{3}} \Gamma_{1} \Rightarrow$ $\Delta_{1}$. There are the following cases for $\Gamma_{1} \mapsto \Delta_{1}$.

Case 1. $\Gamma_{1} \mapsto \Delta_{1}=\Gamma_{2}, A_{1} \wedge A_{2} \mapsto \Delta_{2} \in \xi$. Then, $\Gamma_{1} \mapsto \Delta_{1}$ has a direct child node $\Gamma_{2}, A_{1}, A_{2} \mapsto \Delta_{2}$ (that is, $\left.\Gamma_{2}, A_{1} \mapsto \Delta_{2} \Gamma_{2}, A_{2} \mapsto \Delta_{2}\right)$. By the assumption, we have

$$
\begin{aligned}
& \vdash_{G_{3}} \Gamma_{2}, A_{1} \mapsto \Delta_{2}, \\
& \vdash_{G_{3}} \Gamma_{2}, A_{2} \mapsto \Delta_{2},
\end{aligned}
$$

and by $\left(\mapsto \wedge^{L}\right)$ in $G_{3}, \vdash_{G_{3}} \Gamma_{2}, A_{1} \wedge A_{2} \mapsto \Delta_{2}$.

Case 2. $\Gamma_{1} \mapsto \Delta_{1}=\Gamma_{2}, A_{1} \vee A_{2} \mapsto \Delta_{2} \in \xi$. Then, $\Gamma_{1} \mapsto \Delta_{1}$ has two direct child node $\Gamma_{2}, A_{1} \mapsto \Delta_{2}$ and $\Gamma_{2}, A_{2} \mapsto \Delta_{2}$. There is an $i \in\{1,2\}$ such that $\Gamma_{2}, A_{i} \mapsto$ $\Delta_{2} \in \xi$. By the induction assumption, we have

$$
\vdash_{G_{3}} \Gamma_{2}, A_{1} \mapsto \Delta_{2},
$$

and by $\left(\mapsto \vee^{L}\right)$ in $G_{3}, \vdash_{G_{3}} \Gamma_{2}, A_{1} \vee A_{2} \Rightarrow \Delta_{2}$.

Case 3. $\Gamma_{1} \mapsto \Delta_{1}=\Gamma_{2} \mapsto B_{1} \wedge B_{2}, \Delta_{2} \in \xi$. Then, $\Gamma_{1} \mapsto \Delta_{1}$ has a direct child node $\Gamma_{2} \mapsto B_{1}, B_{2}, \Delta_{2}$ (that is, $\left.\Gamma_{2} \mapsto B_{1}, \Delta_{2} \Gamma_{2} \mapsto B_{2}, \Delta_{2}\right)$. By the induction assumption,

$$
\begin{aligned}
& \vdash_{G_{3}} \Gamma_{2} \mapsto B_{1}, \Delta_{2}, \\
& \vdash_{G_{3}} \Gamma_{2} \mapsto B_{2}, \Delta_{2},
\end{aligned}
$$

and by $\left(\mapsto \wedge^{R}\right)$ in $G_{3}, \vdash_{G_{3}} \Gamma_{2} \mapsto B_{1} \wedge B_{2}, \Delta_{2}$.

Case 4. $\Gamma_{1} \mapsto \Delta_{1}=\Gamma_{2} \mapsto B_{1} \vee B_{2}, \Delta_{2} \in \xi$. Then, $\Gamma_{1} \mapsto \Delta_{1}$ has two direct child nodes $\Gamma_{2} \mapsto B_{1}, \Delta_{2}$ and $\Gamma_{2} \mapsto B_{2}, \Delta_{2}$. There is an $i \in\{1,2\}$ such that $\Gamma_{2} \mapsto$ $B_{i}, \Delta_{2} \in \xi$. By the assumption, we have

$$
\vdash_{G_{3}} \Gamma_{2} \mapsto B_{i}, \Delta_{2},
$$

and by $\left(\mapsto \vee_{i}^{R}\right)$ in $G_{3}, \vdash_{G_{3}} \Gamma_{2} \mapsto B_{1} \vee B_{2}, \Delta_{2}$.

Theorem 5.14 If each leaf $\Gamma^{\prime} \mapsto \Delta^{\prime}$ of $T$ is not an axiom in $G_{3}$ then $T$ is a proof tree of $\Gamma \Rightarrow \Delta$ in $G^{3}$.

Proof. Directly from the definition of $T$.

\subsection{The nonmonotonicity of $G_{3}$}

Theorem 5.15 (The monotonicity theorem). $G^{3}$ is monotonic in both $\Gamma$ and $\Delta$, that is, for any formula sets $\Gamma, \Gamma^{\prime}, \Delta$ and $\Delta^{\prime}$

$$
\begin{aligned}
& \Gamma \subseteq \Gamma^{\prime} \& \vdash_{G^{3}} \Gamma \mapsto \Delta \text { implies } \vdash_{G^{3}} \Gamma^{\prime} \mapsto \Delta ; \\
& \Delta \subseteq \Delta^{\prime} \& \vdash_{G^{3}} \Gamma \mapsto \Delta \text { implies } \vdash_{G^{3}} \Gamma \mapsto \Delta^{\prime} .
\end{aligned}
$$

Theorem 5.16 (The nonmonotonicity theorem). $G_{3}$ is nonmonotonic in both $\Gamma$ and $\Delta$, that is, for any formula sets

ISSN 1927-6974 E-ISSN 1927-6982 


\section{$\Gamma, \Gamma^{\prime}, \Delta$ and $\Delta^{\prime}$,}

$$
\begin{aligned}
& \Gamma \subseteq \Gamma^{\prime} \& \vdash_{G_{3}} \Gamma \mapsto \Delta \text { may not imply } \vdash_{G_{3}} \Gamma^{\prime} \mapsto \Delta ; \\
& \Delta \subseteq \Delta^{\prime} \& \vdash_{G_{3}} \Gamma \mapsto \Delta \text { may not imply } \vdash_{G_{3}} \Gamma \mapsto \Delta^{\prime} .
\end{aligned}
$$

Proof. We prove that the axiom is nonmonotonic and each deduction rule preserves the monotonicity.

Assume that $\operatorname{con}(\Gamma), \operatorname{con}(\neg \Delta)$ and $\Gamma \cap \neg \Delta=\emptyset$. There is a superset $\Gamma^{\prime} \supseteq \Gamma$ such that $\Gamma^{\prime} \cap \neg \Delta \neq \emptyset$; and there is a superset $\Delta^{\prime} \supseteq \Delta$ such that $\Gamma \cap \neg \Delta^{\prime} \neq \emptyset$. Hence, $G_{3}$ is nonmonotonic in both $\Gamma$ and $\Delta$.

To show that $\left(\mapsto \wedge^{R}\right)$ preserves the monotonicity of $\Gamma$, assume that $\Gamma \mapsto B_{1}, \Delta$ and $\Gamma \mapsto B_{2}, \Delta$ are monotonic with respect to $\Gamma$. By $\left(\mapsto \wedge^{R}\right)$, from $\Gamma \mapsto B_{1}, \Delta$ and $\Gamma \mapsto B_{2}, \Delta$, we infer $\Gamma \mapsto B_{1} \wedge B_{2}, \Delta$. Then, for any $\Gamma^{\prime} \supseteq \Gamma, \Gamma^{\prime} \mapsto$ $B_{1}, \Delta$ and $\Gamma^{\prime} \mapsto B_{2}, \Delta$ follows by the assumptions; and by $\left(\mapsto \wedge^{R}\right)$, from $\Gamma^{\prime} \mapsto B_{1}, \Delta$ and $\Gamma^{\prime} \mapsto B_{2}, \Delta$, we infer $\Gamma^{\prime} \mapsto B_{1} \wedge B_{2}, \Delta$. Hence, $\Gamma \mapsto B_{1} \wedge B_{2}, \Delta$ implies $\Gamma^{\prime} \mapsto B_{1} \wedge B_{2}, \Delta$, that is, $\Gamma \mapsto B_{1} \wedge B_{2}, \Delta$ is monotonic with respect to $\Gamma$.

To show that $\left(\mapsto \wedge^{R}\right)$ preserves the nonmonotonicity of $\Gamma$, assume that $\Gamma \mapsto B_{1}, \Delta$ and $\Gamma \mapsto B_{2}, \Delta$ are nonmonotonic with respect to $\Gamma$. By $\left(\mapsto \wedge^{R}\right)$, from $\Gamma \mapsto B_{1}, \Delta$ and $\Gamma \mapsto B_{2}, \Delta$, we infer $\Gamma \mapsto B_{1} \wedge B_{2}, \Delta$. Then, for some $\Gamma^{\prime} \supseteq \Gamma$

$$
\begin{aligned}
& \Gamma \mapsto A_{1}, \Delta \text { may not imply } \Gamma^{\prime}, A_{1} \not \mapsto \Delta ; \\
& \Gamma \mapsto A_{2}, \Delta \text { may not imply } \Gamma^{\prime}, A_{2} \not \mapsto \Delta ;
\end{aligned}
$$

and by $\left(\mapsto \wedge^{R}\right), \Gamma \mapsto B_{1} \wedge B_{2}, \Delta$ may not imply $\Gamma^{\prime} \mapsto$ $B_{1} \wedge B_{2}, \Delta$, that is, $\Gamma \mapsto B_{1} \wedge B_{2}, \Delta$ is nonmonotonic with respect to $\Gamma$.

To show that $\left(\mapsto \wedge^{R}\right)$ preserves the monotonicity of $\Delta$, assume that $\Gamma \mapsto B_{1}, \Delta$ and $\Gamma \mapsto B_{2}, \Delta$ are monotonic with respect to $\Delta$. By $\left(\mapsto \wedge^{R}\right)$, from $\Gamma \mapsto B_{1}, \Delta$ and $\Gamma \mapsto B_{1}, \Delta$, we infer $\Gamma \mapsto B_{1} \wedge B_{2}, \Delta$. Then, for any $\Delta^{\prime} \supseteq \Delta, \Gamma \mapsto$ $B_{1}, \Delta^{\prime}$ and $\Gamma \mapsto B_{2}, \Delta$ follows; and by $\left(\mapsto^{\wedge}\right)$, from $\Gamma \mapsto B_{1}, \Delta$ and $\Gamma \mapsto B_{2}, \Delta$, we infer $\Gamma \mapsto B_{1} \wedge, \Delta^{\prime}$. Hence, $\Gamma \mapsto B_{1} \wedge B_{2}, \Delta$ implies $\Gamma \mapsto B_{1} \wedge B_{2}, \Delta^{\prime}$, that is, $\Gamma \mapsto B_{1} \wedge B_{2}, \Delta$ is monotonic with respect to $\Delta$.

To show that $\left(\mapsto \wedge^{R}\right)$ preserves the nonmonotonicity of $\Delta$, assume that $\Gamma \mapsto B_{1}, \Delta$ and $\Gamma \mapsto B_{2}, \Delta$ are nonmonotonic with respect to $\Delta^{\prime}$. By $\left(\mapsto \wedge^{R}\right)$, from $\Gamma \mapsto B_{1}, \Delta$ and $\Gamma \mapsto B_{2}, \Delta$, we infer $\Gamma \mapsto B_{1} \wedge B_{2}, \Delta$. Then, for some $\Delta^{\prime} \supseteq \Delta$,

$$
\begin{aligned}
& \Gamma \mapsto B_{1}, \Delta \text { may not imply } \Gamma \mapsto B_{1}, \Delta^{\prime} ; \\
& \Gamma \mapsto B_{2}, \Delta \text { may not imply } \Gamma \mapsto B_{2}, \Delta^{\prime} ;
\end{aligned}
$$

and by $\left(\mapsto \wedge^{R}\right), \Gamma \mapsto B_{1} \wedge B_{2}, \Delta$ may not imply $\Gamma \mapsto$ $B_{1} \wedge B_{2}, \Delta^{\prime}$, that is, $\Gamma \mapsto B_{1} \wedge B_{2}, \Delta$ is nonmonotonic with respect to $\Delta$.

Similar to show that other deduction rules preserve the monotonicity and nonmonotonicity with respect to $\Gamma$ and $\Delta$.

By the soundness and completeness theorem, we have that for any formula sets $\Gamma, \Gamma^{\prime}, \Delta$ and $\Delta^{\prime}$,

$$
\begin{aligned}
& \Gamma \subseteq \Gamma^{\prime} \& \models_{G_{3}} \Gamma \mapsto \Delta \text { may not imply } \models_{G_{3}} \Gamma^{\prime} \mapsto \Delta, \\
& \Delta \subseteq \Delta^{\prime} \& \models_{G_{3}} \Gamma \mapsto \Delta \text { may not imply } \models_{G_{3}} \Gamma \mapsto \Delta^{\prime} .
\end{aligned}
$$

\section{The Propositional Logic $G^{4}$}

Definition 6.1 A sequent $\Gamma \Rightarrow \Delta$ is $G^{4}$-valid, denoted by $\models_{G^{4}} \Gamma \Rightarrow \Delta$ if for any assignment $v, v \models \Gamma$ implies $v \models \Delta$, where $v \models \Gamma$ if for every $A \in \Gamma, v(A)=1$; and $v \models \Delta$ if for each $B \in \Delta, v(B)=0$.

Proposition 6.2 Let $\Gamma, \Delta$ be sets of literals. $\models_{G^{4}} \Gamma \Rightarrow \Delta$ if and only if

$$
\neg \Delta \subseteq \Gamma \text { or } \operatorname{incon}(\Gamma) .
$$

Proof. Assume that $\neg \Delta \subseteq \Gamma \operatorname{rorincon}(\Gamma)$. Then, $\models_{G^{4}} \Gamma \Rightarrow$ $\Delta$.

Conversely, assume that $\neg \Delta \nsubseteq \Gamma$ and $\operatorname{con}(\Gamma)$. There is a literal $l \in \neg \Delta-\Gamma$. Define an assignment $v$ such that for any propositional variable $p$,

$v(p)= \begin{cases}1 & \text { if } p \in \Gamma \\ 0 & \text { if } \neg p \in \Gamma \\ 0 & \text { if } p=l \\ 1 & \text { if } p=\neg l \\ 0 & \text { otherwise. }\end{cases}$

Then, $v \models \Gamma$ and $v \not \models \Delta$.

The Gentzen deduction system $G^{4}$ consists of the following axioms and deduction rules:

- Axioms:

$$
\left(A_{\Rightarrow}\right) \frac{\neg \Delta \nsubseteq \Gamma \& \operatorname{con}(\Gamma)}{\Gamma \Rightarrow \neg \Delta},
$$

where $\Delta, \Gamma$ are sets of literals.

- Deduction rules:

$$
\begin{array}{ll}
\left(\Rightarrow \wedge_{1}^{L}\right) \frac{\Gamma, A_{1} \Rightarrow \Delta}{\Gamma, A_{1} \wedge A_{2} \Rightarrow \Delta} & \left(\Rightarrow \wedge_{1}^{R}\right) \frac{\Gamma \Rightarrow B_{1}, \Delta}{\Gamma \Rightarrow B_{1} \wedge B_{2}, \Delta} \\
\left(\Rightarrow \wedge_{2}^{L}\right) \frac{\Gamma, A_{2} \Rightarrow \Delta}{\Gamma, A_{1} \wedge A_{2} \Rightarrow \Delta} & \left(\Rightarrow \wedge_{2}^{R}\right) \frac{\Gamma \Rightarrow B_{2}, \Delta}{\Gamma \Rightarrow B_{1} \wedge B_{2}, \Delta} \\
\left(\Rightarrow \vee^{L}\right) \frac{\Gamma, A_{1} \Rightarrow \Delta \Gamma, A_{2} \Rightarrow \Delta}{\Gamma, A_{1} \vee A_{2} \Rightarrow \Delta} & \left(\Rightarrow \vee^{R}\right) \frac{\Gamma \Rightarrow B_{1}, \Delta \Gamma \Rightarrow B_{2}, \Delta}{\Gamma \Rightarrow B_{1} \vee B_{2}, \Delta}
\end{array}
$$

Theorem 6.3 (The soundness and completeness theorem). 
For any sequent $\Gamma \Rightarrow \Delta$,

$$
\vdash_{G^{4}} \Gamma \Rightarrow \Delta \text { iff } \models_{G^{4}} \Gamma \Rightarrow \Delta .
$$

\section{The propositional logic $G_{4}$}

Definition 6.4 A sequent $\Gamma \mapsto \Delta$ is $G_{4}$-valid, denoted by $\models_{G_{4}} \Gamma \mapsto \Delta$ if there is an assignment $v$ such that $v \models \Gamma$ and $v \models \Delta$, where $v \models \Gamma$ if for every $A \in \Gamma, v(A)=1$; and $v \models \Delta$ if for some $B \in \Delta, v(B)=1.5$.

Proposition 6.5 Let $\Gamma, \Delta$ be sets of literals. $\models_{G_{4}} \Gamma \mapsto \Delta$ if and only if

$$
\neg \Delta \nsubseteq \Gamma \& \operatorname{con}(\Gamma)
$$

The Gentzen deduction system $G_{4}$ consists of the following axioms and deduction rules:

- Axioms:

$$
\left(A_{\mapsto}\right) \frac{\neg \Delta \nsubseteq \Gamma \& \operatorname{con}(\Gamma)}{\Gamma \mapsto \Delta},
$$

where $\Delta, \Gamma$ are sets of literals.

- Deduction rules:

$$
\begin{array}{ll}
\left(\mapsto \wedge^{L}\right) \frac{\Gamma, A_{1} \mapsto \Delta \Gamma, A_{2} \mapsto \Delta}{\Gamma, A_{1} \wedge A_{2} \mapsto \Delta} & \left(\mapsto \wedge^{R}\right) \frac{\Gamma \mapsto B_{1}, \Delta \Gamma \mapsto B_{2}, \Delta}{\Gamma \mapsto B_{1} \wedge B_{2}, \Delta} \\
\left(\mapsto \vee_{1}^{L}\right) \frac{\Gamma, A_{1} \mapsto \Delta}{\Gamma, A_{1} \vee A_{2} \mapsto \Delta} & \left(\mapsto \vee_{1}^{R}\right) \frac{\Gamma \mapsto B_{1}, \Delta}{\Gamma \mapsto B_{1} \vee B_{2}, \Delta} \\
\left(\mapsto \vee_{2}^{L}\right) \frac{\Gamma, A_{2} \mapsto \Delta}{\Gamma, A_{1} \vee A_{2} \mapsto \Delta} & \left(\mapsto \vee_{2}^{R}\right) \frac{\Gamma \mapsto B_{2}, \Delta}{\Gamma \mapsto B_{1} \vee B_{2}, \Delta}
\end{array}
$$

Theorem 6.6 (The soundness and completeness theorem).
For any sequent $\Gamma \mapsto \Delta$,

$$
\vdash_{G_{4}} \Gamma \mapsto \Delta \text { iff } \models G_{4} \Gamma \mapsto \Delta .
$$

\section{Conclusions}

In this paper we proved that $G^{1}, G^{2}, G^{3}, G^{4}, G_{1}, G_{2}, G_{3}, G_{4}$ are sound and complete, and their monotonicity given in the following table:

\begin{tabular}{l|lll} 
system & precondition & mono $\Gamma$ & mono $\Delta$ \\
\hline $\mathbf{G}^{1}$ & incon $(\Gamma) \vee \operatorname{incon}(\Delta) \vee \Gamma \cap \Delta \neq \emptyset$ & $Y$ & $Y$ \\
$\mathbf{G}_{1}$ & $\operatorname{con}(\Gamma) \wedge \operatorname{con}(\Delta) \wedge \Gamma \cap \Delta=\emptyset$ & $N$ & $N$ \\
\hline $\mathbf{G}^{2}$ & $\Delta \subseteq \Gamma \vee \operatorname{incon}(\Gamma)$ & $Y$ & $N$ \\
$\mathbf{G}_{2}$ & $\Delta \nsubseteq \Gamma \wedge \operatorname{con}(\Gamma)$ & $N$ & $Y$ \\
\hline $\mathbf{G}^{3}$ & $\operatorname{incon}(\Gamma) \vee \operatorname{incon}(\neg \Delta) \vee \Gamma \cap \neg \Delta \neq \emptyset$ & $Y$ & $Y$ \\
$\mathbf{G}_{3}$ & $\operatorname{con}(\Gamma) \wedge \operatorname{con}(\neg \Delta) \wedge \Gamma \cap \neg \Delta=\emptyset$ & $N$ & $N$ \\
\hline $\mathbf{G}^{4}$ & $\neg \Delta \subseteq \Gamma \vee \operatorname{incon}(\Gamma)$ & $Y$ & $N$ \\
$\mathbf{G}_{4}$ & $\neg \Delta \nsubseteq \Gamma \wedge \operatorname{con}(\Gamma)$ & $N$ & $Y$
\end{tabular}

where $\neg \Gamma=\{\neg A: A \in \Gamma\}$. Hence, $\neg \Gamma$ is consistent if and only if $\Gamma$ is consistent.

\section{ACKNOWLEDGEMENTS}

This work was supported by the Open Fund of the State Key Laboratory of Software Development Environment under Grant No. SKLSDE-2010KF-06, Beijing University of Aeronautics and Astronautics, and by the National Basic Research Program of China (973 Program) under Grant No. 2005 CB321901.

\section{REFERENCES}

[1] Ebbinghaus H, Flum J, Thomas W. Mathematical Logic, Undergraduate Texts in Mathematics, Berlin, DE/New York, NY: Springer-Verlag, Second Edition; 1994.

[2] Li W. Mathematical Logic: Foundations for Information Science. Progress in Computer Science and Applied Logic. Birkhauser; 2010. http://dx.doi.org/10.1007/978-3-7643-9977-1

[3] Antoniou G. A tutorial on default logics. ACM Computing Surveys. 1994; 31(4): 337-59.

[4] Clark K. Negation as failure. Readings in Nonmonotonic Reasoning. Morgan Kaufmann Publishers. 1987: 311-25.

[5] Reiter R. A logic for default reasoning. Articial intelligence. 1980; 13(1-2): 81-132.

[6] Dahr M. Deductive Databases: Theory and Applications. International Thomson Computer Press; 1997.

[7] Lukaszewicz W. Considerations on default logic: an alternative approach. Computational Intelligence. 1988; 4(1): 1-16. http: //dx.doi.org/10.1111/j.1467-8640.1988.tb00086.x

[8] Marek W, Truszczynski M. Nonmonotonic Logics: ContextDependent Reasoning. Springer. 1993.
[9] Avron A, Lev I. Canonical propositional Gentzen-type systems. IJCAR. 2001

[10] Barwise J. An Introduction to First-Order Logic, in Barwise J (edt.), Handbook of Mathematical Logic. Studies in Logic and the Foundations of Mathematics. Amsterdam. NL: North-Holland; 1982.

[11] Takeuti G. Proof Theory. Amsterdam. NL: North-Holland; 1987.

[12] Delgrande JP, Schaub T, Jackson WK. Alternative approaches to default logic. Articial Intelligence. 1994; 70: 167-237. http://dx .doi.org/10.1016/0004-3702 (94)90106-6

[13] Denecker M, Marek VW, Truszczynski M. Uniform semantic treatment of default and autoepistemic logics. Articial Intelligence. 2003; 143(1): 79-122. http://dx.doi.org/10.1016/S0004-3 702(02) 00293-X

[14] Lloyd JW. Foundations of Logic Programming. Springer-Verlag, Berlin; 1987.

[15] Kaminski M, Rubin-Mosin J. Default theories over monadic languages. Theoretical Computer Science. 2006; 364: 241-53. http: //dx.doi.org/10.1016/j.tcs.2006.08.007 